\title{
Application of Electromagnetic Navigation Bronchoscopy in the early diagnosis and treatment of lung cancer: a narrative review
}

\author{
Wen-Hui Yang $^{1 \#}$, Tian-Qi Xu ${ }^{1 \#}$, Yan Zhang ${ }^{1}$, Lin Jiang ${ }^{2}$, Jian Zhang ${ }^{1}$ \\ ${ }^{1}$ Department of Pulmonary Medicine, Xijing Hospital, Air Force Medical University, Xi'an, China; ${ }^{2}$ Department of Gynecology, Gansu Provincial \\ Maternity and Child-care Hospital, Lanzhou, China \\ Contributions: (I) Conception and design: WH Yang, TQ Xu; (II) Administrative support: J Zhang; (III) Provision of study materials or patients: \\ Y Zhang; (IV) Collection and assembly of data: L Jiang; (V) Data analysis and interpretation: WH Yang, TQ Xu; (VI) Manuscript writing: All \\ authors; (VII) Final approval of manuscript: All authors. \\ "These authors contributed equally to this work \\ Correspondence to: Jian Zhang. Department of Pulmonary Medicine, Xijing Hospital, Air Force Medical University, \#169 West Changle Road, Xi'an \\ 710032, China. Email: 13991802890@163.com.
}

\begin{abstract}
The diagnosis of lung cancer has long been a problem facing clinicians worldwide, and the emergence of electromagnetic navigation bronchoscopy (ENB) has played a critical role in the early diagnosis of lung cancer. Compared with other types of biopsy techniques (e.g., transthoracic needle biopsy, bronchoscopy, thoracoscopic biopsy, and thoracotomy), ENB guarantees high diagnostic accuracy and safety. In recent years, with the continuous development of ENB technology, the scope of its epitaxy has also expanded. This technology is no longer a simple auxiliary diagnosis test but an innovative technology that simultaneously assists in surgical treatment, opening new avenues of research for the treatment of early-stage lung cancer. However, ENB, as a human-mediated operating system, has some limitations and uncertainties in its actual clinical application and promotion, which need to be addressed as we continue to develop ENB technology. In response to the bottleneck in developing ENB technology in current clinical diagnosis and treatment, relevant scientific research and development personnel and clinicians have also performed continuing exploration and improvement of methods. However, to completely overcome the limitations of ENB, more technological innovations are needed. In this review, we describe the current major clinical application directions, application advantages, and limitations of ENB.
\end{abstract}

Keywords: Electromagnetic navigation bronchoscopy (ENB); biopsy; video-assisted thoracoscopic surgery (VATS); radiofrequency ablation (RFA); limitations

Submitted Oct 10, 2020. Accepted for publication Jan 22, 2021.

doi: $10.21037 /$ tcr-20-3020

View this article at: http://dx.doi.org/10.21037/tcr-20-3020

\section{Introduction}

Lung cancer is a leading cause of cancer-related death in China and worldwide (1). Most of the diagnoses of lung cancer occur at advanced stages with a poor prognosis and a 5 -year overall survival rate of $17 \%$. Moreover, patients with an early diagnosis have a 5 -year survival rate of $70 \%$.
These numbers indicate that the early diagnosis of lung cancer is essential for improving the prognosis and survival rate of patients. Patients with early-stage lung cancer usually present with no clear signs or symptoms but solitary pulmonary nodules (SPNs) on chest radiograms. With the increase in public health literacy and advances in chest

\footnotetext{
$\wedge$ ORCID: 0000-0002-4392-5076.
} 
imaging technology, the prevalence of pulmonary nodules has increased in recent years. Notably, the false-positive rate (FPR) of low-dose spiral CT can be as high as $96.4 \%$ (2). That is to say, $96.4 \%$ of the positive screening results in the low-dose CT group were false-positive results. Therefore, precisely diagnosing lung cancer in its early stage has become one of the most urgent tasks facing oncologists (3).

The common invasive diagnostic tests for SPNs include transthoracic needle biopsy, bronchoscopy, thoracoscopic biopsy, and thoracotomy, in which the sensitivity of transthoracic needle biopsy to peripheral malignant nodules can reach 81-97\% (4-6). However, such complications as pneumothorax and bleeding are common in this type of invasive diagnostic test (7). A populationbased study of more than 15,000 patients showed a $15 \%$ incidence of pneumothorax (40\% of patients required chest tube placement) (8). Conventional bronchoscopy is an essential tool for diagnosing lung lesions with a low chance of complications $(<1 \%)$, but its sensitivity toward peripheral nodules is merely $18-62 \%(9,10)$. Although the chest surgical method has high diagnostic accuracy, it has a strong effect on the patient's health status, and wedgeshaped resection or segmental resection of the lung lobe must be performed where the lung nodules are located, usually leading to an adverse effect on the prognosis of the patient. When the pathological result is a benign lesion, contradictions and disagreements between doctors and patients may ensue; thus, pre-surgery assessment of the nodules is vital.

With the evolution of bronchoscopy, its higher safety and yield are receiving increasing attention. Electromagnetic navigation bronchoscopy (ENB) has been used in the diagnosis of clinical SPNs since 2000, and it has been widely used in clinical practice because of the abovementioned characteristics. ENB combines virtual bronchoscopy and electromagnetic localization technology to obtain pathological examination of diseased tissue by transbronchial lung biopsy (TBLB) or transbronchial needle aspiration (TBNA) in real time and accurately through biopsy channels. At the same time, ENB can be used as a surgical auxiliary treatment by positioning the peripheral SPNs or mediastinal and hilar lymph nodes of the lung that cannot be reached by conventional bronchoscopy. In recent years, a new generation of assisted navigation technology represented by the SPiN Thoracic Navigation System has also gradually emerged. Prior to the CT scan, stickers equipped with electromagnetic sensors were placed on the patient's chest and kept in place during the procedure to help guide navigation and track the patient's breathing (Figure 1A,B). Next, a highly accurate 3D map of the lungs that can quickly calculate the shortest path from the lesion and accurately guide the positioning path was synthesized according to the inspiration/expiration CT scanning protocol, providing more strategies for the diagnosis and treatment of peripheral lung lesions.

ENB exhibits a combination of superior diagnostic efficacy, safety, and short positioning time (11-13) and is widely employed in major clinics for the early diagnosis and treatment of lung cancer. However, this technology is subject to a number of limitations and uncertainties in the application process. The following is a summary of the advantages and disadvantages of this technology.

We present the following article in accordance with the Narrative Review reporting checklist (available at http:// dx.doi.org/10.21037/tcr-20-3020).

\section{Methods}

We systematically reviewed 63 research articles and review articles concerning ENB that were published between 1991 to 2021 and combined the system composition, operation methods, and practical applications of ENB in clinical practice. Finally, the bottlenecks and difficulties faced by ENB in the development and promotion process were analyzed based on the actual situation of the diagnosis and treatment of ENB in the current application.

\section{Medical application and safety}

The ENB operating system includes the main body of the tracheal mirror magnetic navigation system, electromagnetic board, navigation positioning catheter, tracheal mirror working channel extension catheter and navigation positioning sensor (Figure 1C,D). To operate $\mathrm{ENB}$, the prior chest thin layer CT image is used for $3 \mathrm{D}$ reconstruction to establish a navigation route, and the guiding catheter is subsequently carried in the bronchoscopy to reach the lesion. Because the guiding catheter tip carries the electromagnetic positioning sensor, the lesion location can be reproduced in real time onto the pre-generated lung $3 \mathrm{D}$ roadmap. The patient lies on the magnetic plate such that the whole chest is in a weak magnetic field, and a special curved catheter with a microsensor inserted into the head end extends into the bronchial cavity. Finally, the catheter can be accurately delivered to the site where the lesion is located for a needle biopsy (Figures 2,3). 

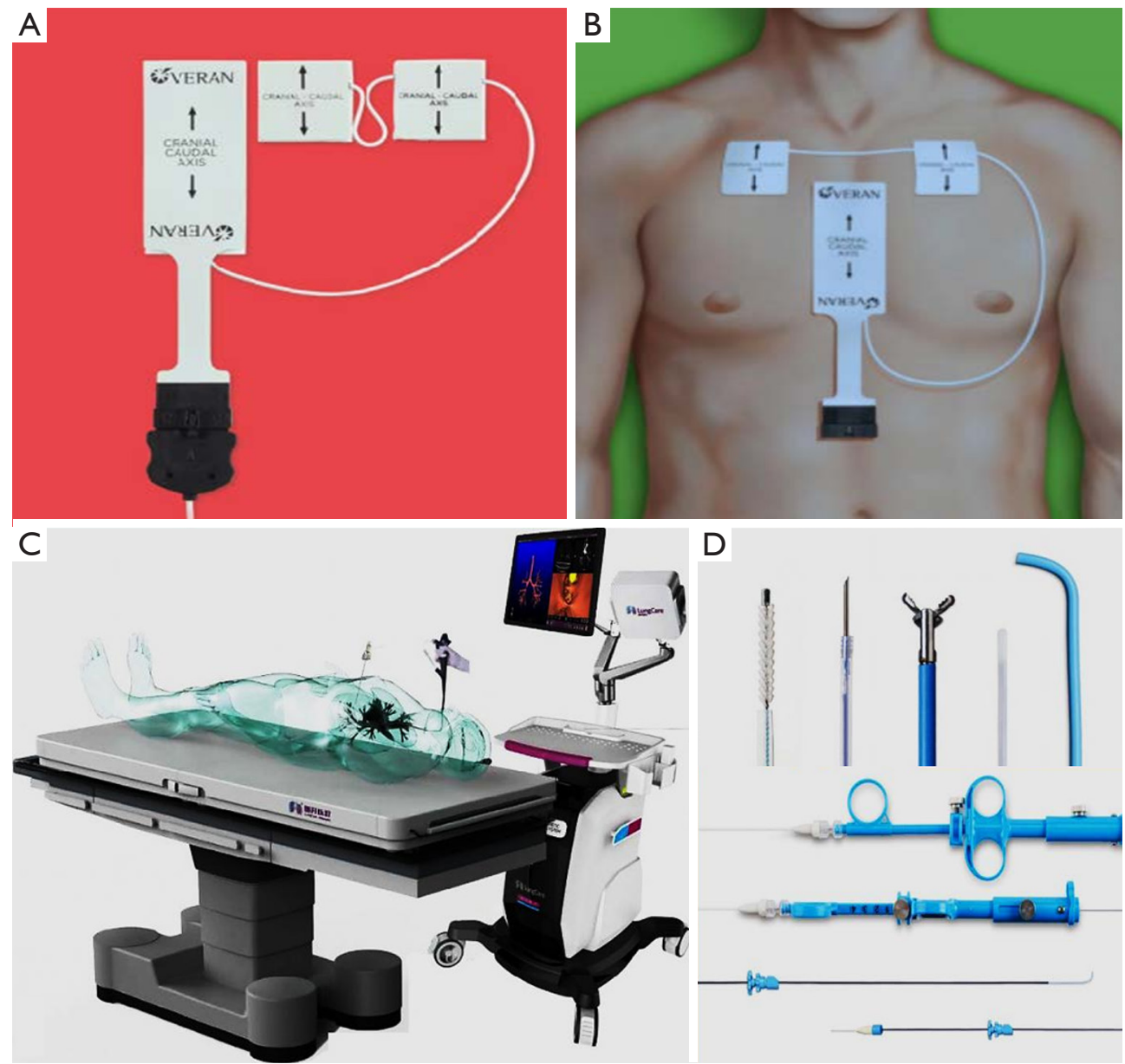

Figure 1 Compositions of ENB operating system. (A) Stickers. (B) Paste method. (C) Tracheal mirror magnetic navigation system, electromagnetic board, navigation positioning catheter, tracheal mirror working channel extension catheter and navigation positioning sensor. (D) Commonly used biopsy devices, including biopsy forceps, biopsy brushes, puncture needles, etc.

\section{Pulmonary nodule biopsy}

Since Solomon et al. (14) first reported the use of ENB in 1998, ENB has been widely used in clinical diagnosis and research. A meta-analysis on the accuracy and safety of ENB in the diagnosis of intrapulmonary nodules in 2014 (10) showed that the sensitivity of ENB for the diagnosis of lung cancer was $71.1 \%$, the negative predictive value was $52.1 \%$, and the incidence of pneumothorax was $3.1 \%, 1.6 \%$ of which required closed thoracic drainage. Another study in 2016 indicated an overall diagnostic yield of ENB of approximately $60 \%$ (15). A prospective multicenter study in 2017 (16) showed that ENB successfully guided 910 subjects (94.4\%) and 1,036 lesions (91.8\%) for biopsy, during which the rates of pneumothorax, bronchopulmonary hemorrhage, and respiratory failure were $4.9 \%$ (grade 2 or higher: $1.0 \%$ ),
$2.3 \%$ (grade 2 or higher: $2.3 \%$ ), and $0.6 \%$, respectively. Another large, multicenter cohort study (17) showed that approximately three-quarters of patients with evaluable lung lesions could be safely diagnosed in all medical institutions and in all challenging areas of the lung. The above findings provide a comprehensive indication that the use of ENB in lung nodule biopsy is safe and effective. Additionally, nodules located in the upper lobe or middle lobe of the right lung, nodules with a long diameter greater than $20 \mathrm{~mm}$, small registration errors, bronchial aeration signs at the lesion site, and lymph node sampling were associated with an increased rate of ENB diagnosis. Additionally, a study has shown that the combined use of the radial scans of the endobronchial ultrasound probe (RP-EBUS) can increase the diagnostic yield of ENB for lung nodules to 


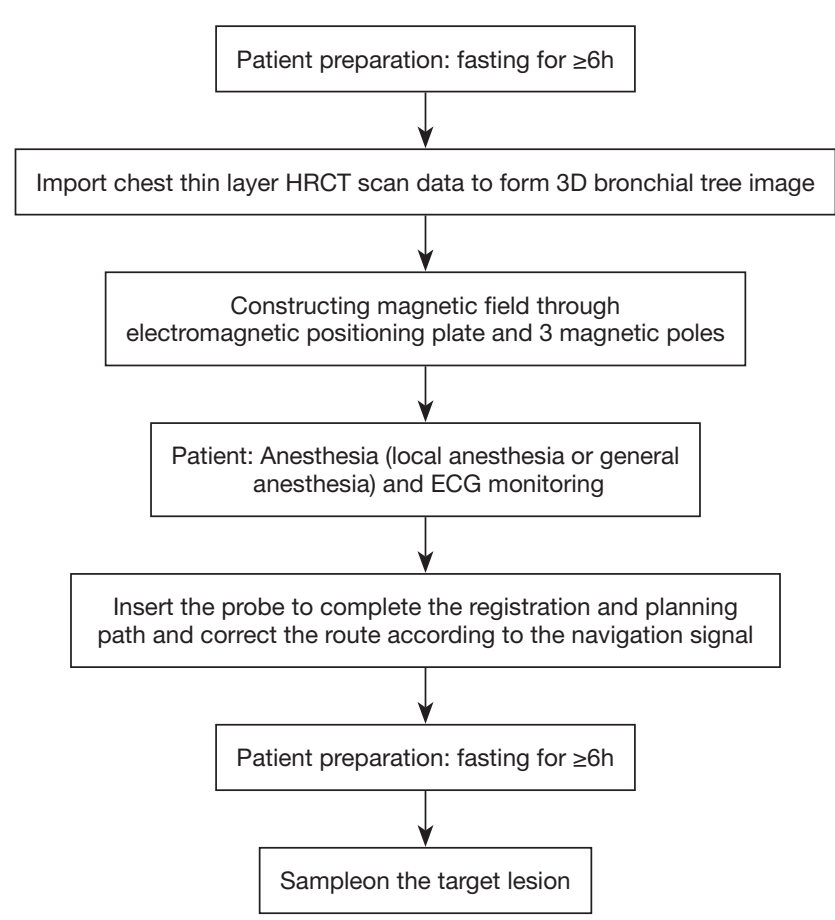

Figure 2 Operation flow of ENB for SPN. HRCT, High-resolution computed tomography; ECG, Electrocardiogram; ENB, electromagnetic navigation bronchoscopy; SPN, solitary pulmonary nodules.

\section{$87.5 \%(18)$.}

To further improve the diagnostic efficacy of ENB, Pritchett et al. (19) performed a biopsy of a pulmonary nodule with a median lesion size of $16.0 \mathrm{~mm}$ (range $7-55 \mathrm{~mm}$ ) using cone beam CT-enhanced fluoroscopy combined with ENB. With a safety margin of $4 \%$, the diagnostic accuracy rate obtained by these researchers reached $93.5 \%$. However, patients using this technique received an average radiation dose of $2.0 \mathrm{mSv}$ each time. Therefore, reducing the radiation dose is very beneficial to patients and doctors. Because the lung tissue has an air-filled alveolar structure, the natural contrast of the lung tissue structure is higher than that of air. Therefore, to a certain extent, reducing the tube current and tube voltage has no pronounced effect on the display and positioning of lung tissue lesions. Additionally, effectively reducing the repeated guidance and positioning of CT is an auxiliary improvement measure to reduce the radiation dose. These measures have effectively reduced the radiation dose and considerably reduced the loss of the tube and detector $(20,21)$.

He et al. (22) showed that the diagnostic yield of ENB guidance cryobiopsy (ENBCB) for lung nodules less than $3.0 \mathrm{~cm}$ was $89.2 \%$. Unfortunately, because of the use of cryosurgery, the complication rate of mild to moderate bleeding was $40.5 \%$. To reduce the occurrence of this complication, the clinic has also made further improvements and provided guidance. First, the freezing time of the probe site must be strictly controlled. Second, cryosurgery should be performed on the lung periphery where the blood vessel density is low. When performing operations on the area close to the hilar where the blood vessel density is high, norepinephrine can be injected into the airway in advance to contract the blood vessels properly. Finally, the balloon can be preinstalled before surgery to prepare for closure. Abundant clinical experience and skilled operation are also important factors in reducing bleeding complications.

\section{Lymph node biopsy}

Accurately determining the stage of early lung cancer is of great significance for treatment options and prognosis. In recent years, with insight into $\mathrm{ENB}$, its diagnostic value and safety for lymph node metastasis and lung cancer staging have also been affirmed. Gildea et al. (23) performed ENB biopsy on enlarged lymph nodes $(28.1 \pm 12.8 \mathrm{~mm})$ in 60 patients for the first time in 2006 and obtained an overall success rate of $100 \%$. A recent study compared the diagnostic value of ENB versus C-TBNA-guided TBNA 


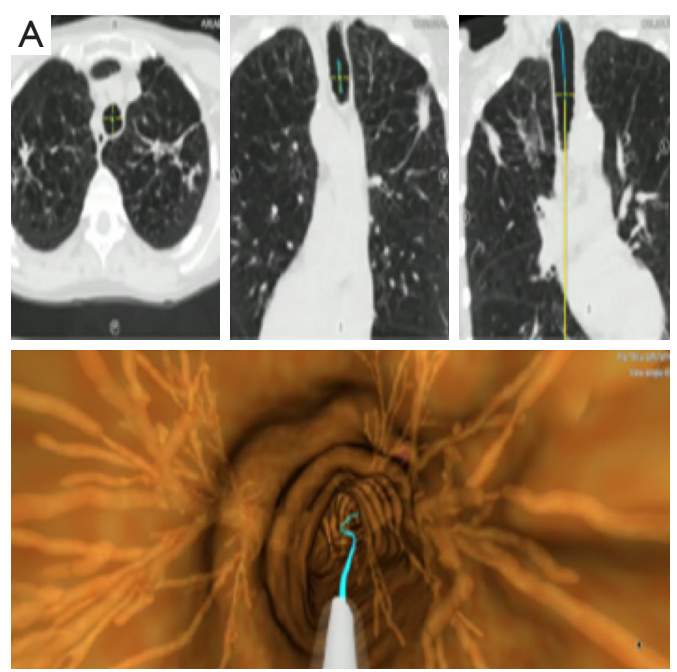

$\mathrm{D}$
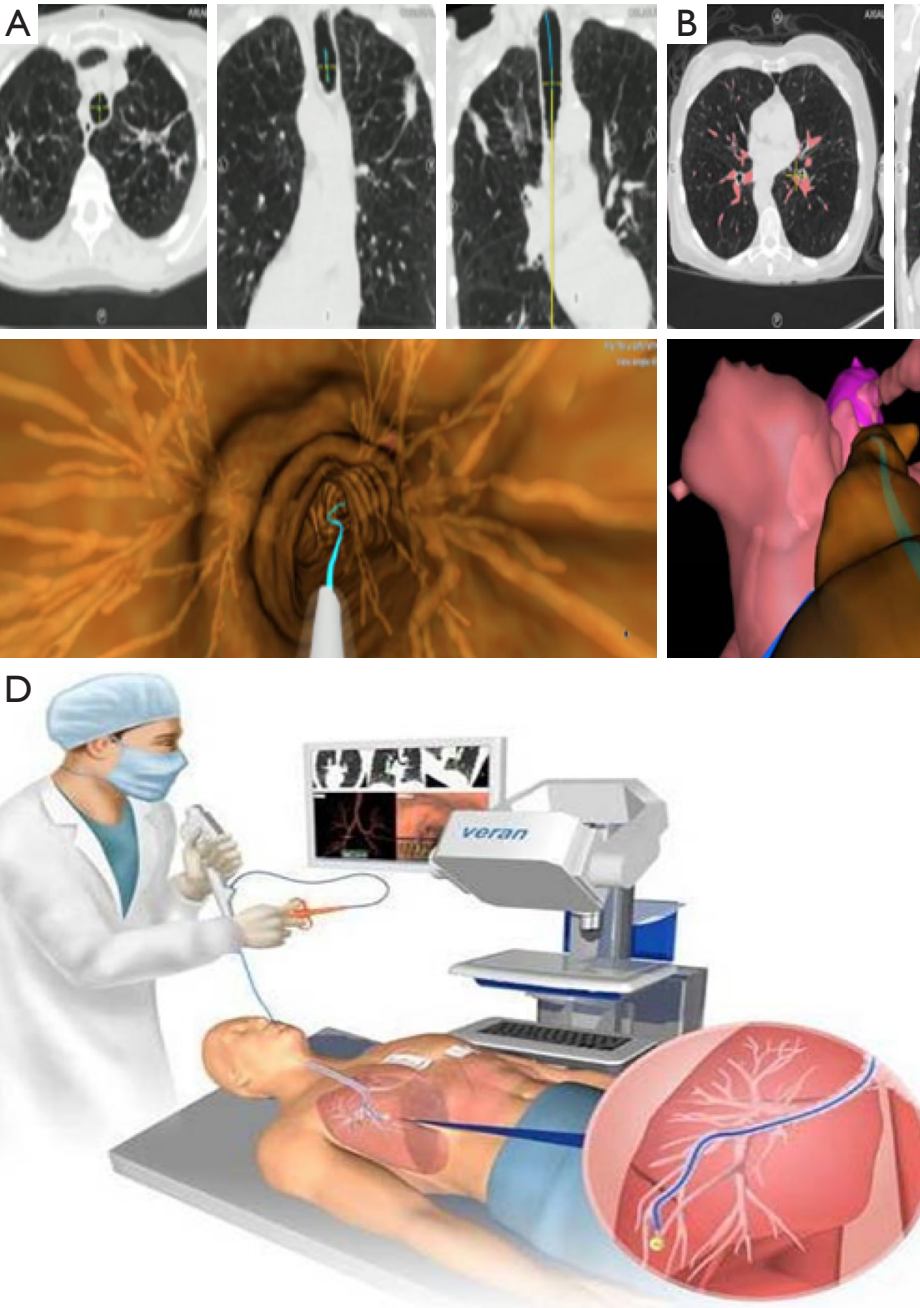
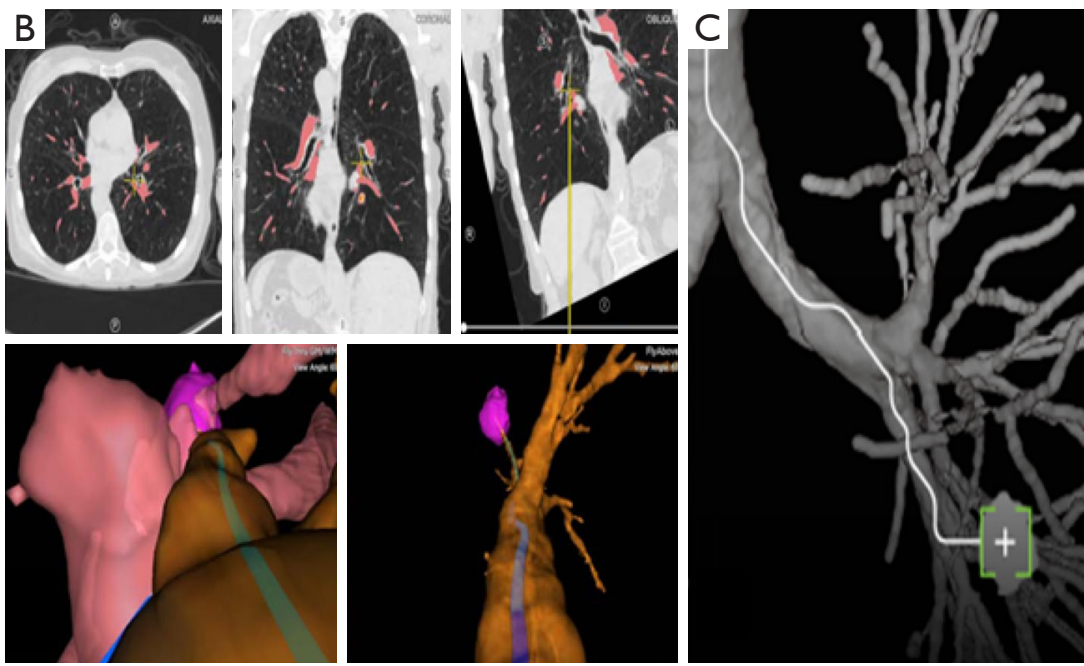

$\mathrm{E}$

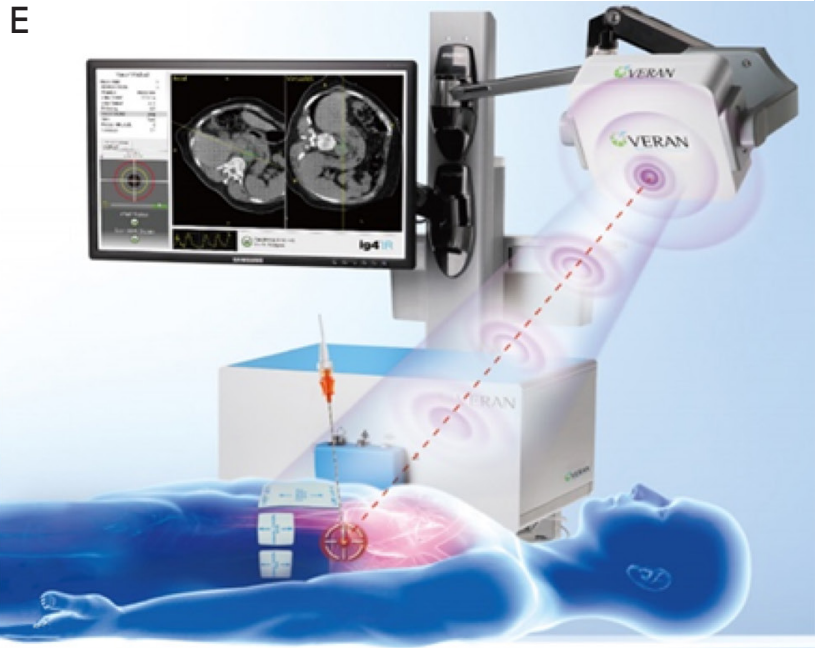

Figure 3 Implementation path of ENB technology. (A) Data collection. (B) Data processing. (C) Preoperative planning and shortest path calculation. (D) Real-time navigation. (E) Location and biopsy of lesions.

in mediastinal and hilar lymphadenopathy. The results showed that the diagnostic yield of ENB-TBNA was $72.8 \%$ compared with only $42.4 \%$ for C-TBNA (24) according to pathological confirmation. ENB combined with rapid on-site evaluation (ROSE) had a success rate of $82.1 \%$ for lymph node biopsies less than $15 \mathrm{~mm}$ and $89.4 \%$ for lymph node biopsies larger than $15 \mathrm{~mm}$ (25).

Moreover, ENB-TBNA also has a greater advantages than EBUS-TBNA. EBUS-TBNA has relatively strict requirements on the location and growth characteristics of lymph nodes. When enlarged lymph nodes are located close to the hilar and grow outside the trachea, EBUS-TBNA can achieve a higher biopsy success rate. However, once there is a tissue partition between the enlarged lymph node and airway, the ultrasound probe cannot detect the lesion well, resulting in a greatly reduced biopsy success rate. ENBTBNA is less affected by the above factors. For swollen lymph nodes that are not adjacent to the hilar, biopsy can still be performed under the conditions of no adherent growth and increased tissue separation from the airway.

In summary, ENB-guided lymph node biopsy not only has a higher diagnostic and sampling rate but also has less restriction and higher safety, during which the complication rate is only $1.2-3.5 \%(23,26)$.

\section{Preoperative positioning of pulmonary nodules}

In the early surgical treatment of lung cancer, thoracic 
surgeons use more video-assisted thoracoscopic surgery (VATS) because of the advantages of smaller trauma, faster postoperative recovery, and safer surgical procedures than open lobectomy $(2,27)$. However, this minimally invasive procedure is often difficult to identify for pulmonary nodules less than $1 \mathrm{~cm}$ during surgery $(28,29)$. Once VATS fails to accurately identify and locate the nodule, it may be temporarily changed to thoracotomy with a risk of surgical resection failure (30). Studies have shown that VATS can result in up to a $46 \%$ conversion rate for thoracotomy in the event of failure to accurately identify lesions (28).

Clinical methods for the localization of small lesions include hookwire localization, coil localization, staining labeling localization and fiducial labeling localization (31-35), in which methylene blue in situ staining does not affect the pathological evaluation of excised specimens (36). Thus, it is more common to use methylene blue to stain the nodules, particularly after positioning under ENB guidance. Methylene blue can mark lung nodules more effectively. Marino et al. (37) recently conducted a large-scale clinical study of methylene blue staining of pulmonary nodules under ENB navigation. These researchers showed a $97.2 \%$ success rate and a $100 \%$ VATS nodule resection rate for a lung nodule with a median size of $8 \mathrm{~mm}$ (range, 4-17 mm). Additionally, no adverse events occur during the marking process under navigation. These results indicate a safer and more effective technique of assisting VATS in removing small pulmonary nodules. The report by Sun et al. (38) described a new method for locating pulmonary nodules with ENB-guided injection of methylene blue combined with percutaneous hookwire localization for patients with positive bronchial signs that was also proven to be safe and effective.

\section{Radiofrequency ablation of lung cancer}

For patients with early-stage non-small-cell lung cancer and partial lung metastases, surgical resection is the preferred treatment (39-41); however, not all patients have physical conditions that meet surgical requirements because of the presence of comorbidities or other contraindications. Therefore, for patients who cannot tolerate surgery, stereotactic radiotherapy or radiofrequency ablation (RFA) is recommended $(42,43)$. Stereotactic radiotherapy is a noninvasive treatment, but its efficacy is restricted because of multiple factors, such as respiratory movements, treatment times, cost and complications of radiation pneumonitis. RFA is a promising alternative therapy with overall survival data showing similar therapeutic effects to subarachnoidectomy and radiation therapy (44-46). However, percutaneous puncture in RFA often causes complications, such as pneumothorax, hemothorax, bronchopleural fistula and pleural effusion, exhibiting an incidence of 15.2-55.6\% (44,47-49). Additionally, percutaneous puncture has difficulty reaching some specific anatomical sites and may cause tumor pleural metastasis or direct spread. However, RFA under bronchoscopy can effectively reduce these serious complications (50). Koizumi et al. (51) demonstrated for the first time that bronchoscopy-guided RFA is a safer and more effective method. Compared with ordinary electronic bronchoscopy, ENB not only can achieve navigation for peripheral lung lesions accurately but can also be used to place therapeutic tools and ablation catheters (52,53). A recent study showed that RFA under ENB navigation is a potentially effective treatment for patients with early-stage lung cancer or lung metastasis. However, limited by the navigation characteristics and heating radius, ENB-guided RFA is only suitable for lesions with a bronchial sign of less than $30 \mathrm{~mm}$ on chest thin-slice CT (54).

\section{Discussion}

The emergence of ENB has considerably improved the diagnostic efficiency of early lung cancer. However, compared with the results of 2007-2018 $(9,15,18,19,25,38,55-58)$, the diagnostic efficiency of ENB alone has not improved significantly in the past 10 years (Table 1). The bottleneck restricting the further improvement of ENB diagnostic efficiency is largely the uncertainty associated with biopsy. Specifically, the objective factors of the patient's lung lesions (e.g., whether there is tracheal access in the lesion, whether the ENB probe can reach the lesion site, whether the lesion is positioned accurately, and the choice of biopsy method) and the subjective factors of ENB operators (e.g., experience, proficiency, correctness of clinical decisionmaking) collectively lead to uncertainty. In the case of ENB combined with other discriminant techniques for the diagnosis of early-stage lung cancer, the safety and effectiveness of the operation cannot be ensured at the same time, except for EBUS and ROSE (18,19,22,25), fully demonstrating the limitations of ENB in the diagnosis of early-stage lung cancer.

First, the location of the SPN has the most important effect on the efficacy rate of ENB. Clinical studies have 
Table 1 Diagnostic yield of ENB for SPN

\begin{tabular}{lcccc}
\hline Study & Number of patients/lesions & Mean diameter mm & Navigate and assistant techniques & Diagnostic yield \% \\
\hline Makris, 2007 (55) & $40 / 40$ & 23.5 & ENB & ENB \\
Eberhardt, 2007 (18) & $39 / 39$ & 28.0 & ENB & 59.5 \\
Bertoletti, 2009 (56) & $54 / 54$ & 31.2 & ENB & 61.1 \\
Al-Jaghbeer, 2016 (15) & $92 / 98$ & 26.0 & ENB+RP-EBUS \\
Eberhardt, 2007 (18) & $40 / 40$ & 24.0 & ENB+ROSE & 60.0 \\
Lamprecht, 2009 (57) & $13 / 13$ & 30.0 & ENB+ROSE & 87.5 \\
Lamprecht, 2012 (58) & $112 / 112$ & 27.1 & ENB+ROSE \\
Karnak, 2013 (25) & $54 / 103$ & 16.5 & ENB+CBCT & 83.9 \\
Pritchett, 2018 (19) & $75 / 93$ & 16.0 & ENBCB & 83.3 \\
He, 2019 (22) & $37 / 37$ & 21.0 & 93.5 \\
\hline
\end{tabular}

ENB, Electromagnetic navigational bronchoscopy; RP-EBUS, Radial probe-endobronchial ultrasound; ROSE, Rapid on-site evaluation; $\mathrm{CBCT}$, Cone-beam computed tomography; ENBCB, Electromagnetic navigation bronchoscopy guidance cryobiopsy.

shown that lesions in the upper and middle lobes of the right lung have higher diagnostic efficiency than other lung lobes using ENB (58). Being affected by the pulsation of the heart and great blood vessels, the left lung has greater mobility than the right lung. At the same time, being affected by respiratory movement, the lower lobe of the lung has greater mobility than the upper lobe (59). These differences can cause ENB navigation and positioning errors, which affect the diagnostic yield. Of course, research on ENB has also made further improvements to reduce errors in clinical applications. On the one hand, the CT data near the examination date (the same day or day before) are used to reconstruct the lung images accurately. On the other hand, a respiratory gating system is introduced based on ENB to restore the patient's accurate image data through respiratory compensation. However, it remains difficult to completely overcome the positioning errors caused by cardiovascular pulsation and respiratory motion. Additionally, studies have shown that the inner two-thirds of the lung using ENB biopsy is twice as large as the peripheral third diagnosis, and the yields in the central, intermediate, and peripheral lesions are $82 \%, 61 \%$, and $53 \%$, respectively $(\mathrm{P}=0.05)$ (9). In addition to the lesions near the periphery of the lung being more affected by respiratory movement, the ability to accurately detect, locate and biopsy the lesions is also an important reason for this phenomenon. In particular, ENB's endoscopic probe cannot reach the airway after level 12 because of its outer diameter and supporting force. Thus, ENB also focuses on developing transparenchymal nodule access (ENB-TPNA) technology in terms of technical exploration. The principle is to make an "artificial tunnel" leading to the lesion in the adjacent airway of the lesion that has no tracheal access or where the probe cannot reach and subsequently biopsy the lesion through this passage. However, the technology is still in the stage of continuous exploration and has not been popularized to date. Second, the size of the SPN is also a major factor affecting the efficacy of ENB. Compared with lesions larger than $20 \mathrm{~mm}$, the diagnostic yield of peripheral lesions less than $20 \mathrm{~mm}$ is lower (60) and has become a consensus in the use of ENB. If ENB positioning technology is combined with percutaneous lung biopsy technology, it can effectively improve the diagnostic performance of lesions with a peripheral diameter of less than $20 \mathrm{~mm}$. Currently, the 4D electromagnetic navigation system developed by related companies is working on this exploration. Third, the location of tumor cells within the SPN directly affects the positive rate of biopsy. Usually, the biopsy tool is located at the geometric center of the lesion. If most of the tumor cells in the lesion are located on the surface or periphery of the lesion, it will affect the accuracy of biopsy. Fourth, the choice of biopsy method, such as bronchoscope needle brushing, bronchial needle aspiration, or bronchial forceps biopsy, can also affect the performance and accuracy of $\operatorname{ENB}(61,62)$.

The clinical practice of ENB and its extensive utilization present several uncertainties that require further consideration. The first is that the diagnostic efficiency of 
the actual clinical application of ENB technology is uneven at each medical center because of discrepancies in skills among ENB inspectors and medical instruments, such as the thin-slice CT scanning technology of the chest. These differences can be decisive in the promotion and extension of ENB. Second, compared with other endoscopy and navigation techniques, ENB has not significantly improved the diagnostic performance of lesions close to the hilar, such as biopsy of hilar enlarged lymph nodes, lesions in the large airways, and other easier-to-reach lesions. It is possible to use ordinary endoscopy techniques to achieve highefficiency diagnostic capabilities. The choice of ENB biopsy strategy at this time will probably increase the burden on patients and the workload of doctors. Therefore, the use of ENB should be considered based on actual conditions. Finally, the choice of local anesthesia and general anesthesia has no significant effect on the diagnostic yield of ENB (63). For the clinical operation of ENB within the specific purposes, physicians must further investigate the selection of anesthesia method to raise the diagnostic yield of lung cancer in the early stage, highlighting the patients' outcomes and their long-term benefits.

\section{Conclusions}

The extensive utilization of ENB has provided an alternative for doctors in the clinical diagnosis and treatment of lung cancer. Because of its higher safety and efficacy, ENB shows unique advantages in the diagnosis and treatment of early-stage lung cancer. It also compensates for blind spots in peripheral lung lesions and minimizes unnecessary complications compared with surgery and standard biopsy. Consistent with advances in recent years, the diagnostic efficiency of ENB has escalated with the combination of various navigation and biopsy techniques, performing as an assistant diagnostic approach in determining lesion locations. Therefore, the promotion of ENB in clinical application helps to elucidate the precise diagnosis of lung cancer in the early stage, and navigationbased treatment also illustrates its potential in performing a precise biopsy for diagnosis and even radical treatment. However, the limitations of this technique cannot be denied. Whether ENB can be used in first-line diagnostic examinations warrants further study.

\section{Acknowledgments}

This manuscript was edited for English language by Matthew B.
Funding: The study is sponsored by National key R \& D plan (2017YFC0112701).

\section{Footnote}

Reporting Checklist: The authors have completed the Narrative Review reporting checklist. Available at http:// dx.doi.org/10.21037/tcr-20-3020

Peer Review File: Available at http://dx.doi.org/10.21037/ tcr-20-3020

Conflicts of Interest: All authors have completed the ICMJE uniform disclosure form (available at http://dx.doi. org/10.21037/tcr-20-3020). The authors have no conflicts of interest to declare.

Ethical Statement: The authors are accountable for all aspects of the work in ensuring that questions related to the accuracy or integrity of any part of the work are appropriately investigated and resolved.

Open Access Statement: This is an Open Access article distributed in accordance with the Creative Commons Attribution-NonCommercial-NoDerivs 4.0 International License (CC BY-NC-ND 4.0), which permits the noncommercial replication and distribution of the article with the strict proviso that no changes or edits are made and the original work is properly cited (including links to both the formal publication through the relevant DOI and the license). See: https://creativecommons.org/licenses/by-nc-nd/4.0/.

\section{References}

1. Ferlay J, Soerjomataram I, Dikshit R, et al. Cancer incidence and mortality worldwide: sources, methods and major patterns in GLOBOCAN 2012. Int J Cancer 2015;136:E359-86.

2. National Lung Screening Trial Research Team, Aberle DR, Adams AM, et al. Reduced lung-cancer mortality with low-dose computed tomographic screening. N Engl J Med 2011;365:395-409.

3. Wu B, Li Y, Hou L, et al. The Diagnostic value of electromagnetic navigation bronchoscopy for pulmonary peripheral nodules. Chinese Journal of Diagnostics (Electronic Edition) 2016;4:203-5.

4. Yao X, Gomes MM, Tsao MS, et al. Fine-needle aspiration biopsy versus core-needle biopsy in diagnosing lung 
cancer: a systematic review. Curr Oncol 2012;19:e16-27.

5. Gould MK, Ananth L, Barnett PG, et al. A clinical model to estimate the pretest probability of lung cancer in patients with solitary pulmonary nodules. Chest 2007;131:383-8.

6. Spiro SG, Gould MK, Colice GL, et al. Initial evaluation of the patient with lung cancer: symptoms, signs, laboratory tests, and paraneoplastic syndromes: ACCP evidenced-based clinical practice guidelines (2nd edition). Chest 2007;132:149S-60S.

7. Gould MK, Fletcher J, Iannettoni MD, et al. Evaluation of patients with pulmonary nodules: when is it lung cancer?: ACCP evidence-based clinical practice guidelines (2nd edition). Chest 2007;132:108S-30S.

8. Wiener RS, Schwartz LM, Woloshin S, et al. Populationbased risk for complications after transthoracic needle lung biopsy of a pulmonary nodule: an analysis of discharge records. Ann Intern Med 2011;155:137-44.

9. Baaklini WA, Reinoso MA, Gorin AB, et al. Diagnostic yield of fiberoptic bronchoscopy in evaluating solitary pulmonary nodules. Chest 2000;117:1049-54.

10. Gex G, Pralong JA, Combescure C, et al. Diagnostic yield and safety of electromagnetic navigation bronchoscopy for lung nodules: a systematic review and meta-analysis. Respiration 2014;87:165-76.

11. Leong S, Ju H, Marshall H, et al. Electromagnetic navigation bronchoscopy: A descriptive analysis. J Thorac Dis 2012;4:173-85.

12. Schwarz Y, Greif J, Becker HD, et al. Real-time electromagnetic navigation bronchoscopy to peripheral lung lesions using overlaid CT images: the first human study. Chest 2006;129:988-94.

13. Mahajan AK, Patel S, Hogarth DK, et al. Electromagnetic navigational bronchoscopy: an effective and safe approach to diagnose peripheral lung lesions unreachable by conventional bronchoscopy in high-risk patients. J Bronchology Interv Pulmonol 2011;18:133-7.

14. Solomon SB, White P Jr, Acker DE, et al. Real-time bronchoscope tip localization enables three-dimensional CT image guidance for transbronchial needle aspiration in swine. Chest 1998;114:1405-10.

15. Al-Jaghbeer M, Marcus M, Durkin M, et al. Diagnostic yield of electromagnetic navigational bronchoscopy. Ther Adv Respir Dis 2016;10:295-9.

16. Khandhar SJ, Bowling MR, Flandes J, et al. Electromagnetic navigation bronchoscopy to access lung lesions in 1,000 subjects: first results of the prospective, multicenter NAVIGATE study. BMC Pulm Med
2017;17:59.

17. Folch EE, Pritchett MA, Nead MA, et al. Electromagnetic Navigation Bronchoscopy for Peripheral Pulmonary Lesions: One-Year Results of the Prospective, Multicenter NAVIGATE Study. J Thorac Oncol 2019;14:445-58.

18. Eberhardt R, Anantham D, Ernst A, et al. Multimodality bronchoscopic diagnosis of peripheral lung lesions: a randomized controlled trial. Am J Respir Crit Care Med 2007;176:36-41.

19. Pritchett MA, Schampaert S, de Groot JAH, et al. ConeBeam CT With Augmented Fluoroscopy Combined With Electromagnetic Navigation Bronchoscopy for Biopsy of Pulmonary Nodules. J Bronchology Interv Pulmonol 2018;25:274-82.

20. Damilakis J. CT Dosimetry: What Has Been Achieved and What Remains to Be Done. Invest Radiol 2021;56:62-8.

21. Sun YJ, Lou J, Xu QL, et al. Comparison of clinical diagnostic value of spiral CT with different dose in patients with early-stage peripheral lung cancer. Clin Transl Oncol 2020. [Epub ahead of print]. doi: 10.1007/s12094-02002503-7.

22. He J, Li XY, Yu M, et al. Electromagnetic navigation bronchoscopy guidance transbronchial lung cryobiopsy for the diagnosis of small peripheral pulmonary lesions. China Journal of Endoscopy 2019;25:54-9.

23. Gildea TR, Mazzone PJ, Karnak D, et al. Electromagnetic navigation diagnostic bronchoscopy: a prospective study. Am J Respir Crit Care Med 2006;174:982-9.

24. Diken ÖE, Karnak D, Ciledag A, et al. Electromagnetic navigation-guided TBNA vs conventional TBNA in the diagnosis of mediastinal lymphadenopathy. Clin Respir J 2015;9:214-20.

25. Karnak D, Ciledag A, Ceyhan K, et al. Rapid on-site evaluation and low registration error enhance the success of electromagnetic navigation bronchoscopy. Ann Thorac Med 2013;8:28-32.

26. Wilson DS, Bartlett RJ. Improved Diagnostic Yield of Bronchoscopy in a Community Practice: Combination of Electromagnetic Navigation System and Rapid On-site Evaluation. J Bronchology Interv Pulmonol 2007;14:227-32.

27. Begum S, Hansen HJ, Papagiannopoulos K. VATS anatomic lung resections-the European experience. J Thorac Dis 2014;6 Suppl 2:S203-10.

28. Suzuki K, Nagai K, Yoshida J, et al. Video-assisted thoracoscopic surgery for small indeterminate pulmonary nodules: indications for preoperative marking. Chest 1999;115:563-8. 
29. Saito H, Minamiya Y, Matsuzaki I, et al. Indication for preoperative localization of small peripheral pulmonary nodules in thoracoscopic surgery. J Thorac Cardiovasc Surg 2002;124:1198-202.

30. McKenna RJ Jr, Houck W, Fuller CB. Video-assisted thoracic surgery lobectomy: experience with 1,100 cases. Ann Thorac Surg 2006;81:421-5; discussion 425-6.

31. Ciriaco P, Negri G, Puglisi A, et al. Video-assisted thoracoscopic surgery for pulmonary nodules: rationale for preoperative computed tomography-guided hookwire localization. Eur J Cardiothorac Surg 2004;25:429-33.

32. Chen S, Zhou J, Zhang J, et al. Video-assisted thoracoscopic solitary pulmonary nodule resection after CT-guided hookwire localization: 43 cases report and literature review. Surg Endosc 2011;25:1723-9.

33. Liu L, Zhang LJ, Chen B, et al. Novel CT-guided coil localization of peripheral pulmonary nodules prior to video-assisted thoracoscopic surgery: a pilot study. Acta Radiol 2014;55:699-706.

34. Andrade RS. Electromagnetic navigation bronchoscopyguided thoracoscopic wedge resection of small pulmonary nodules. Semin Thorac Cardiovasc Surg 2010;22:262-5.

35. Krimsky WS, Minnich DJ, Cattaneo SM, et al. Thoracoscopic detection of occult indeterminate pulmonary nodules using bronchoscopic pleural dye marking. J Community Hosp Intern Med Perspect 2014. doi: 10.3402/jchimp.v4.23084.

36. Zhang Z, Liao Y, Ai B, et al. Methylene blue staining: a new technique for identifying intersegmental planes in anatomic segmentectomy. Ann Thorac Surg 2015;99:238-42.

37. Marino KA, Sullivan JL, Weksler B. Electromagnetic Navigation Bronchoscopy for Identifying Lung Nodules for Thoracoscopic Resection. Ann Thorac Surg 2016;102:454-7.

38. Sun J, Mao X, Xie F, et al. Electromagnetic navigation bronchoscopy guided injection of methylene blue combined with hookwire for preoperative localization of small pulmonary lesions in thoracoscopic surgery. J Thorac Dis 2015;7:E652-6.

39. Scott WJ, Howington J, Feigenberg S, et al. Treatment of non-small cell lung cancer stage I and stage II: ACCP evidence-based clinical practice guidelines (2nd edition). Chest 2007;132:234S-42S.

40. Erhunmwunsee L, D'Amico TA. Surgical management of pulmonary metastases. Ann Thorac Surg 2009;88:2052-60.

41. Friedel G, Pastorino U, Buyse M, et al. Resection of lung metastases: long-term results and prognostic analysis based on 5206 cases--the International Registry of Lung
Metastases. Zentralbl Chir 1999;124:96-103.

42. Gould MK, Donington J, Lynch WR, et al. Evaluation of individuals with pulmonary nodules: when is it lung cancer? Diagnosis and management of lung cancer, 3rd ed: American College of Chest Physicians evidence-based clinical practice guidelines. Chest 2013;143:e93S-e120S.

43. Harzheim D, Eberhardt R, Hoffmann H, et al. The Solitary Pulmonary Nodule. Respiration 2015;90:160-72.

44. Bilal H, Mahmood S, Rajashanker B, et al. Is radiofrequency ablation more effective than stereotactic ablative radiotherapy in patients with early stage medically inoperable non-small cell lung cancer? Interact Cardiovasc Thorac Surg 2012;15:258-65.

45. Dupuy DE, Fernando HC, Hillman S, et al.

Radiofrequency ablation of stage IA non-small cell lung cancer in medically inoperable patients: Results from the American College of Surgeons Oncology Group Z4033 (Alliance) trial. Cancer 2015;121:3491-8.

46. Safi S, Rauch G, op den Winkel J, et al. Sublobar Resection, Radiofrequency Ablation or Radiotherapy in Stage I Non-Small Cell Lung Cancer. Respiration 2015;89:550-7.

47. Zhu JC, Yan TD, Morris DL. A systematic review of radiofrequency ablation for lung tumors. Ann Surg Oncol 2008;15:1765-74.

48. Lanuti M, Sharma A, Digumarthy SR, et al. Radiofrequency ablation for treatment of medically inoperable stage I non-small cell lung cancer. J Thorac Cardiovasc Surg 2009;137:160-6.

49. Tomiyama N, Yasuhara Y, Nakajima Y, et al. CTguided needle biopsy of lung lesions: a survey of severe complication based on 9783 biopsies in Japan. Eur J Radiol 2006;59:60-4.

50. Harris K, Puchalski J, Sterman D. Recent Advances in Bronchoscopic Treatment of Peripheral Lung Cancers. Chest 2017;151:674-85.

51. Koizumi T, Tsushima K, Tanabe T, et al. BronchoscopyGuided Cooled Radiofrequency Ablation as a Novel Intervention Therapy for Peripheral Lung Cancer. Respiration 2015;90:47-55.

52. Anantham D, Feller-Kopman D, Shanmugham LN, et al. Electromagnetic navigation bronchoscopy-guided fiducial placement for robotic stereotactic radiosurgery of lung tumors: a feasibility study. Chest 2007;132:930-5.

53. Harms W, Krempien R, Grehn C, et al. Electromagnetically navigated brachytherapy as a new treatment option for peripheral pulmonary tumors. Strahlenther Onkol 2006;182:108-11. 
54. Xie F, Zheng X, Xiao B, et al. Navigation BronchoscopyGuided Radiofrequency Ablation for Nonsurgical Peripheral Pulmonary Tumors. Respiration 2017;94:293-8.

55. Makris D, Scherpereel A, Leroy S, et al. Electromagnetic navigation diagnostic bronchoscopy for small peripheral lung lesions. Eur Respir J 2007;29:1187-92.

56. Bertoletti L, Robert A, Cottier M, et al. Accuracy and feasibility of electromagnetic navigated bronchoscopy under nitrous oxide sedation for pulmonary peripheral opacities: an outpatient study. Respiration 2009;78:293-300.

57. Lamprecht B, Porsch P, Pirich C, et al. Electromagnetic navigation bronchoscopy in combination with PET-CT and rapid on-site cytopathologic examination for diagnosis of peripheral lung lesions. Lung 2009;187:55-9.

58. Lamprecht B, Porsch P, Wegleitner B, et al. Electromagnetic navigation bronchoscopy (ENB): Increasing diagnostic yield. Respir Med 2012;106:710-5.

Cite this article as: Yang WH, Xu TQ, Zhang Y, Jiang L, Zhang J. Application of Electromagnetic Navigation Bronchoscopy in the early diagnosis and treatment of lung cancer: a narrative review. Transl Cancer Res 2021;10(3):15831593. doi: $10.21037 /$ tcr-20-3020
59. Chen A, Pastis N, Furukawa B, et al. The effect of respiratory motion on pulmonary nodule location during electromagnetic navigation bronchoscopy. Chest 2015;147:1275-81.

60. Wang Memoli JS, Nietert PJ, Silvestri GA. Metaanalysis of guided bronchoscopy for the evaluation of the pulmonary nodule. Chest 2012;142:385-93.

61. Eberhardt R, Morgan RK, Ernst A, et al. Comparison of suction catheter versus forceps biopsy for sampling of solitary pulmonary nodules guided by electromagnetic navigational bronchoscopy. Respiration 2010;79:54-60.

62. Wang KP, Britt EJ. Needle brush in the diagnosis of lung mass or nodule through flexible bronchoscopy. Chest 1991;100:1148-50.

63. Eberhardt R, Anantham D, Herth F, et al. Electromagnetic navigation diagnostic bronchoscopy in peripheral lung lesions. Chest 2007;131:1800-5. 